\title{
Development of tuneable Fabry-Pérot sensors for parallelised photoacoustic signal acquisition
}

\author{
Villringer, Claus, Gilani, Taravat, Zhang, Edward, Pulwer, \\ Silvio, Steglich, Patrick, et al.
}

Claus Villringer, Taravat S. Gilani, Edward Zhang, Silvio Pulwer, Patrick Steglich, Sigurd Schrader, Jan Laufer, "Development of tuneable Fabry-Pérot sensors for parallelised photoacoustic signal acquisition," Proc. SPIE 10878, Photons Plus Ultrasound: Imaging and Sensing 2019, 108780M (8 March 2019); doi: 10.1117/12.2509437

SPIE. Event: SPIE BiOS, 2019, San Francisco, California, United States 


\title{
Development of tuneable Fabry-Pérot sensors for parallelised photoacoustic signal acquisition
}

\author{
Claus Villringer*a,b, Taravat S. Gilani ${ }^{\mathrm{b}}$, Edward Zhang ${ }^{\mathrm{c}}$, Silvio Pulwer ${ }^{\mathrm{a}}$, Patrick Steglich ${ }^{\mathrm{a}, \mathrm{d}}$, \\ Sigurd Schrader ${ }^{\mathrm{a}}$, Jan Laufer ${ }^{\mathrm{b}}$ \\ ${ }^{a}$ Technical University of Applied Sciences Wildau, Germany \\ bInstitut für Physik, Martin-Luther-Universität Halle-Wittenberg, Germany \\ 'Department of Medical Physics and Biomedical Engineering, University College London, UK \\ ${ }^{\mathrm{d} I H P}$ - Leibniz-Institut für innovative Mikroelektronik, Germany
}

\begin{abstract}
Fabry-Pérot (FP) sensors have enabled high resolution 3D photoacoustic (PA) imaging in backward mode. However, raster-scanning of the interrogation laser beam across the sensor can result in slow $3 \mathrm{D}$ image acquisition. To overcome this limitation, parallelized PA signal acquisition can be used for which FP sensors with uniform optical thickness are required. In this work, the optical thickness is tuned a) irreversibly through the use of a photopolymer host matrix and b) actively using embedded electro-optic (EO) chromophores. Polymer spacers $(5 \mu \mathrm{m})$ were deposited using spin coating and sandwiched between two dielectric mirrors and transparent ITO electrodes. The employed polymer guest-host system consists of an EO chromophore (2-methyl-4-nitroaniline) and poly(vinyl cinnamate). EO tuneability was induced using contact poling and a tuneability of $68 \mathrm{pm}$ was demonstrated. The optical thickness was homogenised by raster scanning a UV beam whilst varying the exposure time across a $4 \mathrm{~mm}^{2}$ detection aperture.
\end{abstract}

Keywords: Photoacoustic imaging, Fabry-Pérot polymer film sensors, tuneable Fabry-Pérot

\section{INTRODUCTION}

Fabry-Pérot (FP) polymer film ultrasound sensors offer small element sizes, high acoustic sensitivity, flat frequency response, and transparency to excitation pulses, enabling high resolution 3D photoacoustic (PA) imaging in backward mode [1,2]. PA waves modulate the optical thickness of the FP sensor which results in a change in the reflected optical power. This is conventionally measured using an interrogation laser beam focused on the FP sensor and the PA wave field is mapped by raster-scanning the interrogation beam across the sensor. Due to the spatial variation of the spacer thickness, the interrogation wavelength has to be tuned at each scan point to ensure the maximum phase sensitivity. However, raster-scanning can result in slow 3D image acquisition. To overcome this limitation, novel parallelized readout schemes such as the use of multiple interrogation beams [3] and a camera-based setup [4] have been investigated. An advantage of the camera-based setup are potential improvements in the optical phase sensitivity due to the use of a collimated interrogation beam [5]. This approach requires a sufficiently uniform thickness of the polymer spacer over the scan area to obtain high acoustic sensitivity for a large number of active elements. The challenge lies in the deposition of polymer-based spacers with high homogeneity of thickness. In order to compensate the fabricationinduced inhomogeneity in the layer thickness, we propose to correct the refractive index of the polymer spacer locally, which is shown schematically in figure 1 . The optical thickness is tuned a) irreversibly through the use of a photopolymer host matrix and UV illumination, and b) actively, using embedded electro-optic (EO) chromophores. An optical thickness correction is made with the photopolymers and while the EO effect can subsequently be used to fine tune the optical thickness of the spacer and to compensate thermal drifts during PA measurements.

*claus.villringer@th-wildau.de; phone +49 3375508770

Photons Plus Ultrasound: Imaging and Sensing 2019, edited by Alexander A. Oraevsky, Lihong V. Wang, Proc. of SPIE Vol. 10878, 108780M - (C) 2019 SPIE · CCC code: 1605-7422/19/\$18 · doi: 10.1117/12.2509437 

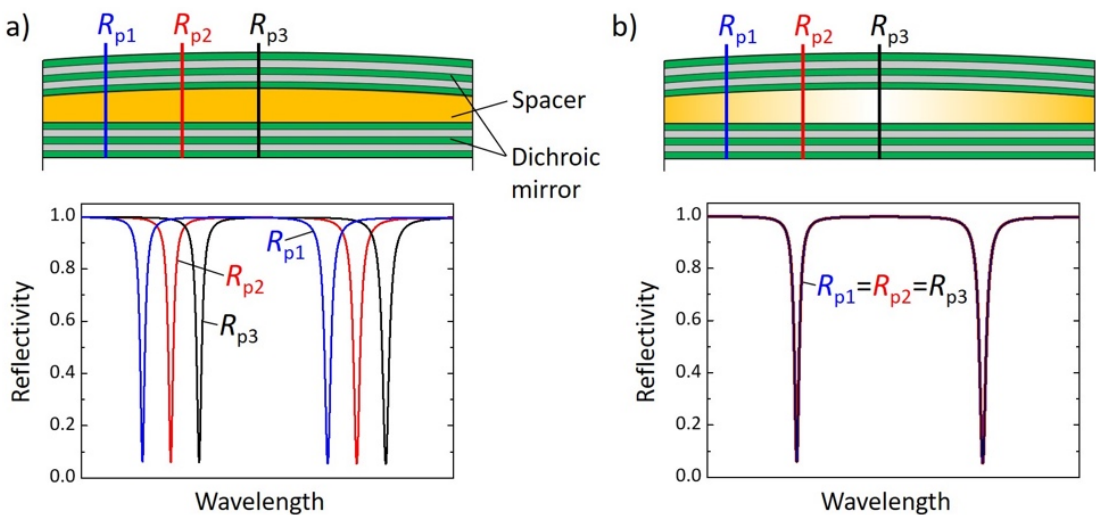

Figure 1. Illustration of the correction of the FP optical thickness. a) Spectra of the reflectivity at different sensor points vary due to the inhomogeneity of the spacer thickness. b) Optical thickness homogenisation can be achieved through a local change in the refractive index.

\section{BACKGROUND}

\subsection{Refractive index change of polymers}

EO polymers offer an actively controlled and reversible change the refractive index. They consist of active dipolar molecules, also called EO chromophores, which are embedded in a passive polymer matrix. Linear EO activity requires a macroscopic non-centrosymmetric orientation which is usually achieved by electric field poling. In this case, the polymer is heated above to the glass transition temperature and an electric field is applied to which the EO molecules are then aligned. After cooling the material to room temperature while the electric field is kept on, the molecules remain oriented. If an electric field is now applied to the poled EO polymer, the refractive index changes linearly and reversibly as a function of the applied voltage.

For an irreversible correction of the refractive index, photopolymers can be used. Photochemical reactions in polymer films can induce various changes such as transparency, thickness, and refractive index [6]. Poly(vinyl cinnamate) (PVCi) is a suitable photopolymer in which cinnamoyl groups photodimerize under UV irradiation $[6,7]$. During photodimerization, the loss of $\pi$-conjugation from a benzene ring to a carbonyl group leads to a large change in electronic delocalisation, with a large change in the refractive index [8].

\section{METHODS}

\subsection{Spacer material}

The EO photopolymer spacer material must be transparent in the excitation band between $600 \mathrm{~nm}$ and $1600 \mathrm{~nm}$ and highly transparent in the interrogation band between $500 \mathrm{~nm}$ and $532 \mathrm{~nm}$. Even minor optical absorption has a detrimental effect on FP finesse and thus acoustic sensitivity. 2-Methyl-4-nitroaniline (MNA) was used as EO chromophore since the absorption maximum of this chromophore is located at about $380 \mathrm{~nm}[9,10]$. This leads to a relative high linear EO effect in the visible spectra range [9]. As a host polymer, PVCi was used due to the large refractive index tuneability [8]. The final guest-host system, which was a mixture of the EO chromophore and PVCi, contained $10 \mathrm{wt} \%$ MNA. The refractive index and extinction coefficient of the used guest-host system is shown in figure 2. The interrogation band was chosen due to a) negligible absorption, b) the high EO coefficient and c) the excitation band. 


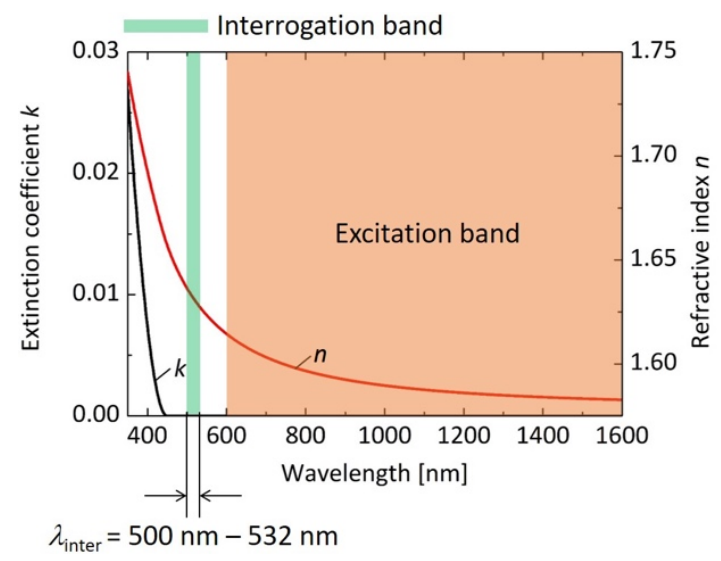

Figure 2. Refractive index and extinction coefficient of the EO photopolymer spacer material PVCi $+10 \mathrm{wt} \% \mathrm{MNA}$.

\subsection{Sensor fabrication}

A transparent indium tin oxide (ITO) electrode and a dichroic mirror consisting of alternating layers of the dielectric materials silicon dioxide $\left(\mathrm{SiO}_{2}\right)$ and titanium dioxide $\left(\mathrm{TiO}_{2}\right)$ were deposited on a glass substrate using reactive magnetron sputtering. The refractive index of these layers are $n_{\mathrm{SiO} 2}=1.48$ and $n_{\mathrm{TiO} 2}=2.49$ at $\lambda=516 \mathrm{~nm}$. The dichroic mirror with the transparent electrode shows a high transmission in the entire excitation band and a high reflectivity in the interrogation band. The EO photopolymer spacer with a thickness of about $5 \mu \mathrm{m}$ was deposited using spin coating. The samples were placed in a vacuum oven at $70^{\circ} \mathrm{C}$ for about $72 \mathrm{~h}$ to remove the solvent. After that, the second dichroic mirror and electrode was deposited. $600 \mathrm{~V}$ were applied on the electrodes at a temperature of $50^{\circ} \mathrm{C}$ for contact poling the EO photopolymer spacer. Figure 3a shows a schematic of the sensor.

Next, FP sensors with photopolymer spacer were fabricated. For this the same deposition methods as for the FP sensor with the EO photopolymer spacer were used. In this case, the electrodes were omitted as illustrated in figure $3 \mathrm{~b}$.
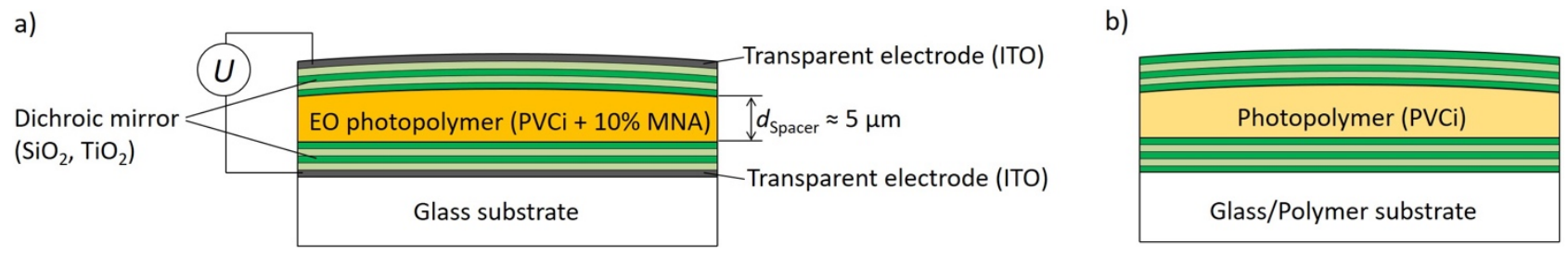

Figure 3. Schematic of the FP sensors with a) EO photopolymer spacer and b) photopolymer spacer.

\subsection{Optical thickness homogenisation}

The sensor was positioned on a motorised XY stage. Visible light was focused on the FP sensor (spot size $\approx 170 \mu \mathrm{m}$ ) and the reflectivity was measured point by point (step size $=50 \mu \mathrm{m}$ ) using a spectrometer (HR4000, Ocean Optics). To determine the fringe position, a Lorentzian function was fitted to the measured interferometer transfer function of the FP sensor. To homogenise the spacer thickness, the sensor was exposed point-by-point to UV light from a xenon arc lamp. After exposure, the shift of the fringes was measured by rescanning the sensor using the spectrometer. Figure 4 shows a schematic of the employed setup. 


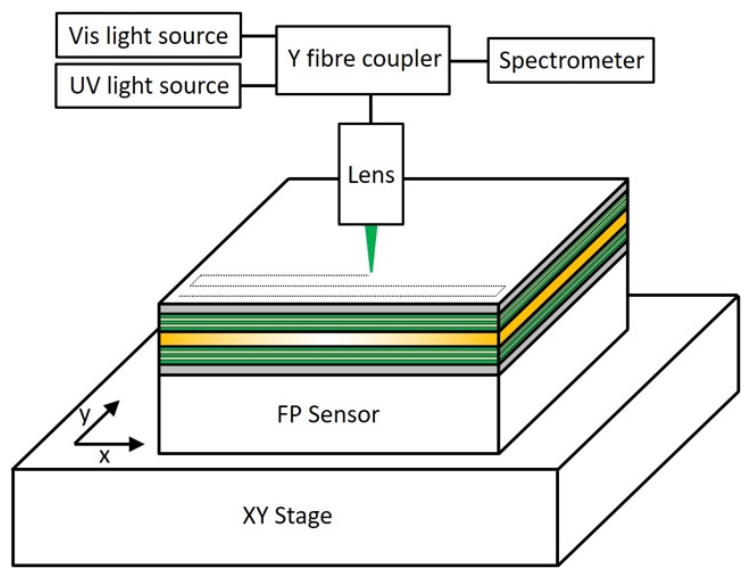

\subsection{EO tuneability}

Figure 4. Setup for the optical thickness homogenisation.

Before measuring the EO tuneability, a part of the sensor was exposed using a xenon arc lamp and a long-pass filter with a cut-on wavelength of $420 \mathrm{~nm}$. The filter is necessary to avoid bleaching of the EO chromophores. The shift of the fringes was measured by applying an electrical voltage between $0 \mathrm{~V}$ and $600 \mathrm{~V}$ to the electrodes and measuring the reflectivity point by point using the setup shown in figure 4 .

\section{RESULTS}

\subsection{Optical thickness homogenisation}

Figure 5a shows the shift of the fringes as a function of the sensor position for two different exposure times. In the exposed areas a homogeneous fringe shift can be seen. The exposure time of the edges of the exposed areas are different due to the overlap of the raster scan. This lead to a transition of the fringe shift, which can be seen in figure $5 \mathrm{~b}$. Based on measurements with different exposure times, a calibration curve was determined. This calibration curve provides the exposure time depending on the intended fringe shift and was used for the homogenisation of the spacer.

a)

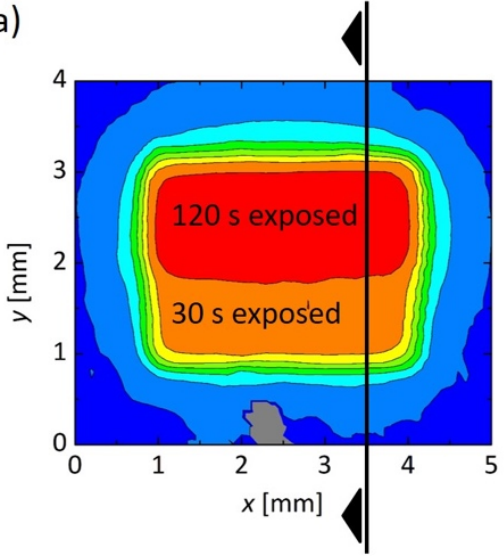

b)

Fringe shift $\Delta \lambda[\mathrm{nm}]$

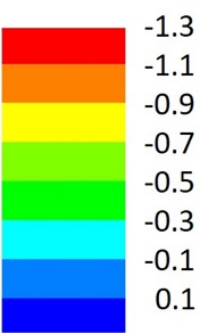

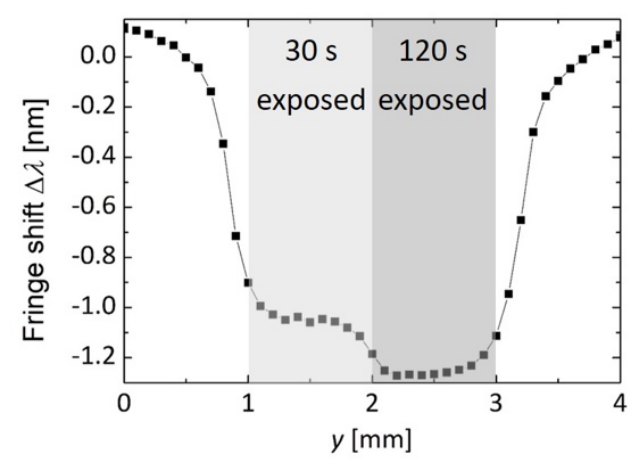

Figure 5. Shift of the fringes for two different exposure times as a function of the sensor position a) $\mathrm{x}$ and $\mathrm{y}$ and $\mathrm{b}$ ) $\mathrm{y}$.

Figure 6 shows the fringe position of a small sensor area before (figure 6a) and after (figure 6b) the homogenisation of the spacer. 
a)

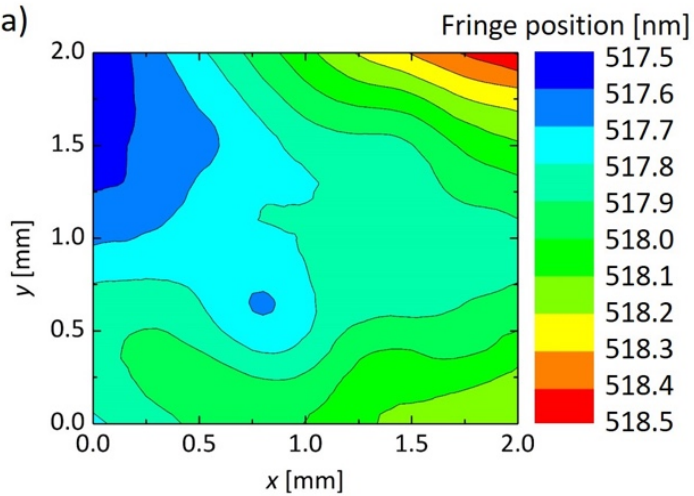

b)

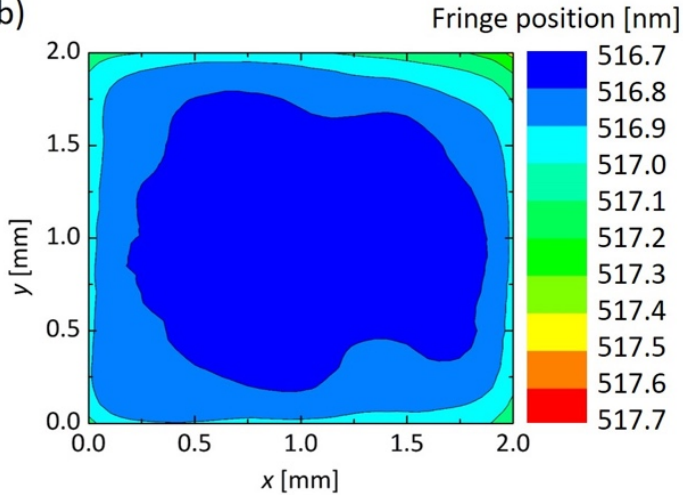

Figure 6. Fringe position of a small sensor area a) before and b) after homogenising the spacer.

\subsection{EO tuneability}

Figure 7a shows the fringe position of a partial area of the sensor, with the left side exposed and the right side unexposed. In the exposed area, a shift of the fringes to smaller wavelengths can be seen. Figure $7 \mathrm{~b}$ shows the fringe shift as a function of the applied electric field. A reversible fringe shift of $\Delta \lambda=-68 \mathrm{pm} \pm 2 \mathrm{pm}$ for the exposed area and $\Delta \lambda=-79 \mathrm{pm} \pm 3 \mathrm{pm}$ for the unexposed area was determined by applying $600 \mathrm{~V}$. The average values of the areas shown in figure $7 \mathrm{a}$ were used to determine the fringe shift.

a)

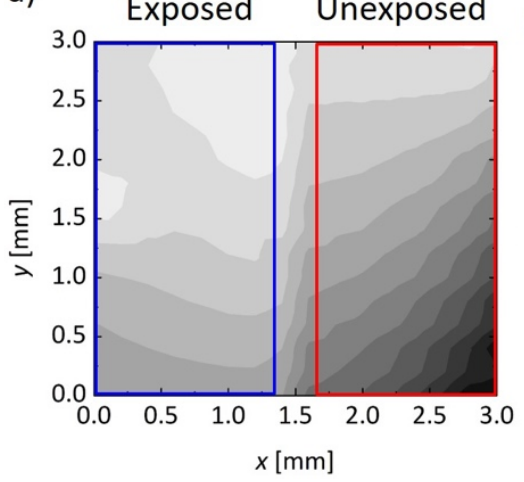

b) Fringe position [nm]

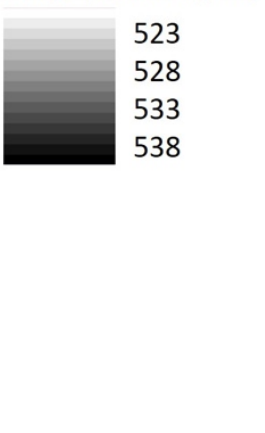

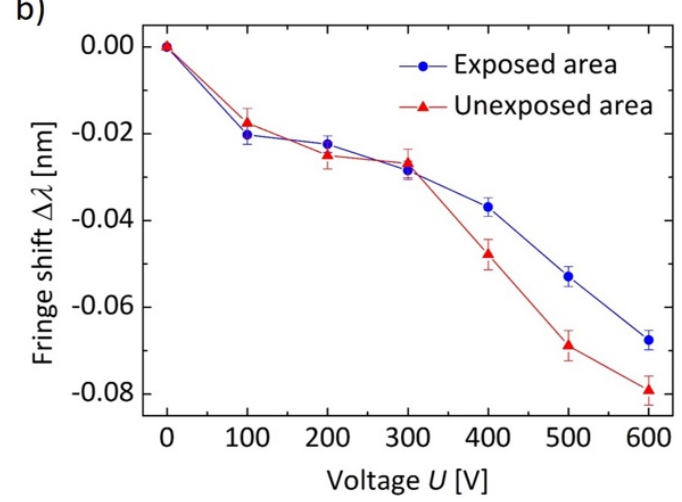

Figure 7. a) Fringe position of a sensor with exposed und unexposed area and b) fringe shift as a function of the applied electric field.

\section{DISCUSSION AND CONCLUSIONS}

Optically transparent tuneable FP sensors based on EO photopolymer spacer were fabricated. EO photopolymer spacers have been shown to allow active optical thickness fine tuning using the EO effect and the photopolymer allows for optical thickness correction of typical spin coated FP spacers. The relatively low EO tuneability can be improved by optimising the electrical poling process, increasing the chromophore concentration and by using advanced EO chromophores. Tuneable FP sensors have the potential to enable parallel PA signal acquisition using a camera-based setup.

\section{ACKNOWLEDGEMENTS}

This work was funded by the German Research Foundation under contract number 624657. 


\section{REFERENCES}

[1] Zhang, E., Laufer, J. and Beard, P., "Backward-mode multiwavelength photoacoustic scanner using a planar Fabry-Perot polymer film ultrasound sensor for high-resolution three-dimensional imaging of biological tissues," Appl. Opt. 47(4), 561-577 (2008).

[2] Jathoul, A. P., Laufer, J., Ogunlade, O., Treeby, B., Cox, B., Zhang, E., Johnson, P., Pizzey, A. R., Philip, B., Marafioti, T., Lythgoe, M. F., Pedley, R. B., Pule, M. a. and Beard, P., "Deep in vivo photoacoustic imaging of mammalian tissues using a tyrosinase-based genetic reporter," Nat. Photonics, 9(4), 239-246 (2015).

[3] Huynh, N., Ogunlade, O., Zhang, E., Cox, B. and Beard P., "Photoacoustic imaging using an 8-beam FabryPerot scanner," Proc. SPIE 9708, 97082L (2016).

[4] Gilani, T. S., Villringer, C., Zhang, E., Gundlach, H., Buchmann, J., Schrader, S. and Laufer, J., "Parallelised photoacoustic signal acquisition using a Fabry-Perot sensor and a camera-based interrogation scheme," Proc. SPIE 10494, 1049431 (2018).

[5] Guggenheim, J. A., Li, J., Allen, T. J., Colchester, R. J., Noimark, S., Ogunlade, O., Parkin, I. P., Papakonstantinou, I., Desjardins, A. E., Zhang, E. Z. and Beard, P. C., "Ultrasensitive plano-concave optical microresonators for ultrasound sensing," Nat. Photonics, 11(11), 714 (2017).

[6] Reiser, A., [Photoreactive Polymers], Wiley-Interscience, New York (1989).

[7] Reiser, A. and Egerton, P. L., "Mechanism of crosslink formation in solid polyvinylcinnamate and related photopolymers," Photogr. Sci. Eng. 23, 144 (1979).

[8] Murase, S., Kinoshita, K., Horie, K. and Morino S., "Photo-optical Control with Large Refractive Index Changes by Photodimerization of Poly(vinyl cinnamate) Film," Macromolecules 30(25), 8088 (1997).

[9] Lipscomb, G. F., Garito, A. F. and Narang, R. S., "An exceptionally large linear electro-optic effect in the organic solid MNA," J. Chem. Phys, 75(3), 1509 (1981).

[10] Morita, R., Ogasawara, N., Umegaki, S. and Ito, R., "Refractive indices of 2-methyl-4-nitroaniline (MNA)," Jpn. J. Appl. Phys, 26(10A), L1711 (1987). 